\title{
Effectiveness of Metformin on Weight Loss in Non-Diabetic Individuals with Obesity
}

Authors

Affiliations

\author{
C. Seifarth ${ }^{1}$, B. Schehler ${ }^{1}$, H. J. Schneider ${ }^{2}$
}

${ }^{1}$ Practice for Endocrinology, Regensburg, Germany

${ }^{2}$ Medizinische Klinik und Poliklinik IV, Ludwig-Maximilian-University, Munich, Germany
Key words

metformin

obesity

insulin resistance received 09.07.2012 first decision 13.09.2012 accepted $\quad 12.10 .2012$

\section{Bibliography}

Dol http://dx.doi.org/

10.1055/s-0032-1327734

Published online:

November 12, 2012

Exp Clin Endocrinol Diabetes

2013; 121: 27-31

(C) J. A. Barth Verlag in

Georg Thieme Verlag KG

Stuttgart · New York

ISSN 0947-7349

Correspondence

PD Dr. med. C. Seifarth

Weichser Weg 5

93059 Regensburg

Germany

Fax: +49/0941/5989 308

seifarth@hotmail.com

\section{Abstract}

$\nabla$

Objective: The efficacy of metformin for the treatment of obesity has been evaluated in few clinical trials with inconclusive results. Moreover, the effectiveness in a real-life outpatient setting has not been tested until today. In this study we aimed to examine the effectiveness of metformin as a weight reducing drug in obese and overweight patients with regard to their degree of insulin resistance.

Design and patients: We treated 154 consecutive patients with a body mass index $\geq 27 \mathrm{~kg} / \mathrm{m}^{2}$ in an outpatient setting over 6 months with metformin up to a dosage of $2500 \mathrm{mg}$ per day. Additionally, we included 45 untreated patients as controls. Patients were monitored for weight

\section{Introduction}

$\nabla$

Currently, there are only few weight-loss drugs with a favourable side-effect profile available [1] Drugs like rimonabant have been promising in the recent past. However, severe side effects lead to their witdrawal from the market [2]. Presently, GLP-1 analogues such as Liraglutide are being promoted as a new strategy to loose weight without major side effects however at high costs [3].

Metformin is a well established anti-diabetic drug, which has been used from the late $1950 \mathrm{~s}$ on and has since made an unrattled career as an antidiabetic drug which is easy to handle and still first line therapy in type 2 diabetes $[4,5]$. In the last decade it underwent a second career as efficacious treatment of polycystic ovary syndrome (PCOS). Metformin reduces insulin resistance which is the underlying cause of both type 2 diabetes and PCOS $[6,7,25,26]$. Metformin has been observed to cause weight loss in type 2 diabetes patients $[8,9]$. Obesity in non-diabetic patients is also linked to insulin resistance [10-12]. Improving insulin sensitivity may therefore account for changes over 6 months. Before metformin treatment was started insulin sensitivity was determined in all patients by calculating HOMA index and Matsuda index after a $75 \mathrm{~g}$ oral glucose tolerance test.

Results: The mean weight loss in the metformin treated group was $5.8 \pm 7.0 \mathrm{~kg}(5.6 \pm 6.5 \%)$. Untreated controls gained $0.8 \pm 3.5 \mathrm{~kg}(0.8 \pm 3.7 \%)$ on average. Patients with severe insulin resistance lost significantly more weight as compared to insulin sensitive patients. The percentage of weight loss was independent of age, sex or BMI. Conclusion: Metformin is an effective drug to reduce weight in a naturalistic outpatient setting in insulin sensitive and insulin resistant overweight and obese patients.

weight reduction under metformin therapy, although the exact underlying pathomechanisms remain to be elucidated [9-14].

Only one randomized controlled trial of high quality (Diabetes Prevention Program) has been published that shows metformin to significantly reduce weight in non-diabetic patients [15], this effect endured up to 8 years, if patients adhered to the medication [16].

However, results are still inconclusive to date. In 2 further placebo-controlled studies, no significant weight-loss of metformin was found $[17,18]$. Even in very recently published reviews on antiobesity drugs metformin is not mentioned $[19,20]$. In another review metformin is listed as a weight reducing drug, but the scarcity of these studies is underlined [21]. In adults metformin has merely been looked upon as an anti-diabetic drug and significant weight loss under metformin has been described more as a welcome side effect in diabetic and prediabetic patients [22-24]. Interestingly however, for special indications like obese psychiatric patients $[25,26]$ or obese children and adolescents $[27,28]$ as well as 
for women suffering from PCOS [29-31] weight reduction by metformin has been well documented.

Moreover, while the efficacy of metformin to reduce weight in a standardized setting has been tested in few randomized trials, data on effectiveness in the everyday endocrine outpatient setting are still lacking. The aim of this study was to test the effectiveness of metformin to reduce weight in obese, non-diabetic patients in a naturalistic outpatient setting.

\section{Subjects \\ $\nabla$}

In our Practice for Endocrinology in Regensburg, Germany, an outpatient setting for endocrine patients, we screened overweight and obese patients desiring to loose weight for insulin resistance during a recruitment period of 18 months. Patients were offered metformin therapy when their body mass index was $\geq 27 \mathrm{~kg} / \mathrm{m}^{2}$. Insulin sensitivity was evaluated by calculating HOMA- and Matsuda-index after conducting an oral glucose tolerance test (OGTT). We recommended all patients to reduce carbohydrate consumption in the evening, but none of the patients underwent a concomitant diet program. We chose that study design because a diet program would confound the results of the study as a consequence of varying compliance and adherence to the diet. Furthermore, we did not include patients who were planning to change their lifestyle during the observation period, especially regarding physical activity.

Patients suffering from overt diabetes or impaired glucose tolerance or already taking anti-diabetic drugs (including metformin) were excluded. Patients under steroid or antipsychotic medication were also excluded. We further excluded patients suffering from depression or any form of drug addiction including alcoholism and severe smoking (more than one package per day). All patients were euthyroid. Pregnant and nursing women were excluded. At baseline, weight, height, blood pressure, HbA1c, liver enzymes, cholesterol and triglyzerides were measured. Patients were then treated with metformin. The dosage was slowly uptitrated starting with $500 \mathrm{mg}$ per day during the first week, dosage was then weekly increased by $500 \mathrm{mg}$ daily up to the final dose. Patients with a BMI $<30 \mathrm{~kg} / \mathrm{m}^{2}$ received $1500 \mathrm{mg}$ final dose per day, patients with a BMI $\geq 30 \mathrm{~kg} / \mathrm{m}^{2}$ but $<35 \mathrm{~kg} / \mathrm{m}^{2}$ received $2000 \mathrm{mg}$ and patients with a BMI $\geq 35 \mathrm{~kg} / \mathrm{m}^{2} 2500 \mathrm{mg}$. 5 patients did not tolerate the projected dose of metformin due to side effects and were excluded from the study. These 5 patients are not part of the 154 metformin-treated patients analyzed for effectiveness. However, these 5 patients were included in the analyses of adverse events. Therefore, 159 metformintreated patients were analyzed for adverse events.

Patients, who chose not to take a medication as a mean to reduce weight served as a control group. All patients were followed-up for 6 months. All patients agreed to refrain from diet programs as well from a change of their habits in physical excercise for the time of the study and were regularly questioned on that issue.

\section{Methods}

$\nabla$

All patients with a BMI of $\geq 27 \mathrm{~kg} / \mathrm{m}^{2}$ were offered to participate. Prior to inclusion, a $75 \mathrm{~g}$ oral glucose tolerance test was performed in all subjects. Blood glucose as well as plasma insulin levels were measured at baseline, $1 \mathrm{~h}$, and $2 \mathrm{~h}$ after glucose ingestion. Matsuda-Index and HOMA-Index was calculated from measured
Table 1 Baseline clinical characteristics, Means and standard deviation of metabolic parameters are shown.

\begin{tabular}{|lccl|}
\hline & Metformin $(\mathbf{n = 1 5 4})$ & Control (n=45) & p-value \\
\hline age [years] & $37.8 \pm 12.9$ & $40.3 \pm 11.4$ & 0.09 \\
\hline BMI [kg/m2] & $35.4 \pm 6.5$ & $34.9 \pm 4.0$ & 0.92 \\
\hline hypertension [\%] & $20.4 \%$ & $22.2 \%$ & 0.42 \\
\hline systol BP [mmHg] & $122.8 \pm 15.6$ & $123.5 \pm 12$ & 0.89 \\
\hline diast BP [mmHg] & $81.0 \pm 10.5$ & $78.4 \pm 9.6$ & 0.66 \\
\hline cholesterol [mg/dl] & $208 \pm 35$ & $217 \pm 39.5$ & 0.11 \\
\hline HDL-C [mg/dl] & $60 \pm 13.0$ & $61 \pm 25.1$ & 0.59 \\
\hline Triglycerides [mg/dl] & $161 \pm 35.3$ & $154 \pm 32.1$ & 0.08 \\
\hline HbA1c [\%] & $5.6 \pm 0.6$ & $5.7 \pm 0.4$ & 0.9 \\
\hline
\end{tabular}

insulin and blood glucose values [32]. Patients were included in the study when blood glucose levels in the performed OGTT were within the normal range irrespective of the degree of insulin sensitivity. Insulin resistance was established if HOMA index was $>2.5$ or if Matsuda index was $<6$. Insulin was measured by a chemoluminescence assay (Roche, Mannheim, Germany). Blood glucose was measured by Hemocue 201 RT (glucose-dehydrogenase method, HemoCue, Großostheim, Germany).

We analyzed changes in weight after 6 months in metformin treated patients and untreated controls. We additionally calculated the percentage of patients losing at least $5 \%$ and $10 \%$ of their baseline weight, respectively. We then divided baseline BMI, age, C-peptide levels, HOMA-index, and Matsuda-index in tertiles and analyzed weight loss according to the respective tertiles. Additionally we analyzed sex-specific weight loss.

\section{Statistics}

$\nabla$

Significance of weight loss after 6 months of treatment as compared to initial body weight was calculated by using the independent t-test. ANOVA was used to compare the weight loss between subgroups. Mann-Whithney-U-Test was used to compare weight loss between males and females, as well as to compare weight changes in treated and untreated patients. All calculations were performed with WINSTAT for Excel.

\section{Results}

$\nabla$

A total of 199 consecutive patients were included in the study (179 women, 20 men). The average age was 39.2 years, the medium BMI was $35.3 \mathrm{~kg} / \mathrm{m}^{2} \pm 6.0$. A total of 154 patients (138 women, 16 men) were included in the metformin group and 45 patients (41 women, 4 men) were included in the control group. $\odot$ Table 1 displays the clinical baseline characteristics of both groups. There were no differences in age, BMI, blood pressure, lipids and HbA1c. Metformin treatment was in general well tolerated by most of the patients.

The average dose of metformin was $2230 \mathrm{mg}$ daily in the metformin-treated group. 24 (15\%) of patients under metformin complained about gastrointestinal side effects like diarrhea, bloating and stomach pain. In these patients we increased the dosage less rapidely, so that they reached the final dose later but all within 2 month.

We observed a weight loss of $10 \%$ or more in $16.2 \%$ and a weight loss of at least $5 \%$ in $47.4 \%$ of the 154 metformin-treated patients. 
Table 2 Weight loss in $\mathrm{kg}$ and \% of baseline weight by 3 tertiles of Matsuda-Index, C-peptide HOMA-index,age and BMI. Data are presented as mean \pm standard deviation.

\begin{tabular}{|c|c|c|c|c|}
\hline & Tertile I & Tertile II & Tertile III & p-value \\
\hline Matsuda-Index & $>3.5$ & $2.1-3.4$ & $<2.1$ & \\
\hline kg & $5.0 \pm 8.2$ & $6.1 \pm 7.4$ & $6.4 \pm 4.8$ & $p=0.005$ \\
\hline$\%$ & $5.0 \pm 7.8$ & $6.0 \pm 6.9$ & $6.0 \pm 4.2$ & $p=0.0002$ \\
\hline C-peptide & $<3.0$ & $3.0-4,4$ & $>4.5$ & \\
\hline kg & $6.0 \pm 6.6$ & $6.2 \pm 7.8$ & $5.9 \pm 6.5$ & $p=0.12$ \\
\hline$\%$ & $5.9 \pm 5.8$ & $5.5 \pm 7.3$ & $5.6 \pm 6.3$ & $p=0.57$ \\
\hline HOMA & $<3,0$ & $3,0-4,5$ & $>4.5$ & \\
\hline kg & $5.1 \pm 8.5$ & $6.0 \pm 6.7$ & $6.4 \pm 5.4$ & $p=0.004$ \\
\hline$\%$ & $5.1 \pm 8.1$ & $5.9 \pm 6.0$ & $5.9 \pm 5.0$ & $p=0.001$ \\
\hline Age & $<31.5$ & $31.6-42$ & $43-72$ & \\
\hline kg & $5.6 \pm 6.6$ & $6.1 \pm 6.9$ & $5.7 \pm 7.5$ & $p=0.18$ \\
\hline$\%$ & $5.4 \pm 7.0$ & $6.2 \pm 5.7$ & $5.2 \pm 7.1$ & $p=0.09$ \\
\hline BMI & $27-32.6$ & $>32,6-37.4$ & $37.5-50$ & \\
\hline kg & $3.4 \pm 4.9$ & $5.6 \pm 6.6$ & $8.5 \pm 8.2$ & $P=0.02$ \\
\hline$\%$ & $4.1 \pm 5.9$ & $5.7 \pm 6.6$ & $6.9 \pm 6.8$ & $p=0.07$ \\
\hline
\end{tabular}

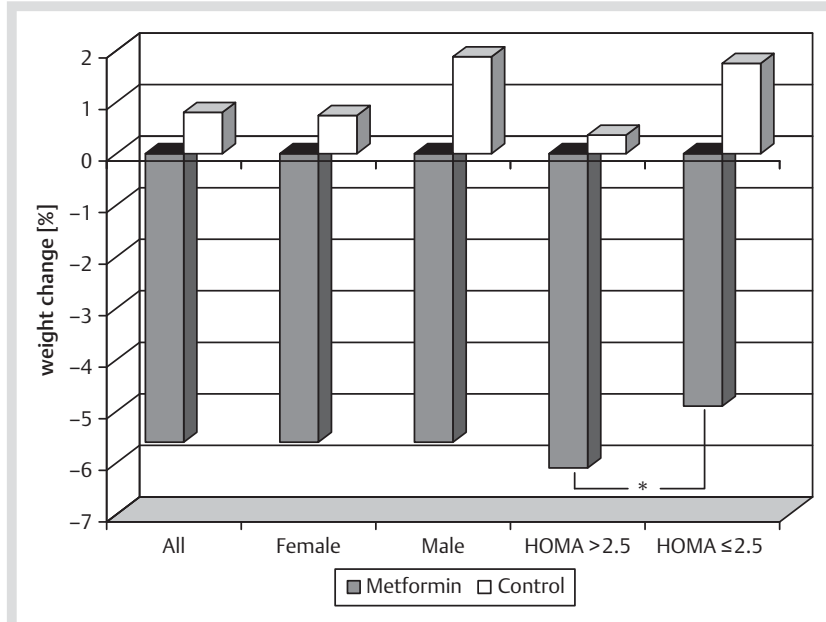

Fig. 1 Weight changes in body weight in relation to base line weight with regard to sex and insulin sensitivity after 6 month of metformin treatment. Insulin resistant patients (HOMA >2.5, $\mathrm{n}=111$ ) lost significantly $\left({ }^{*}\right)$ more weight as compared to patients with normal HOMA-index $(n=43)(p=0.001)$.

In the group metformin-treated, the overall average weight loss during 6 month of treatment was $5.8 \pm 7.0 \mathrm{~kg}(5.6 \pm 6.5 \%)$ $(\mathrm{p}<0.001)$. The range was from the loss of $35 \mathrm{~kg}$ in one patient to a weight gain of $13 \mathrm{~kg}$ in another patient, the median was a weight reduction of $5 \mathrm{~kg}$. 32 patients out of the total of 154 treated patients $(20.8 \%)$ did not loose weight, 9 of them even gained weight under metformin. The weight loss in the metformin treated group was significant as compared to the control group ( $\mathrm{p}<0.0001)$. In the control group an average weight gain of $0.8 \pm 3.5 \mathrm{~kg}(0.8 \pm 3.8 \%)$ was observed.

There was no significant difference in weight loss under metformin treatment between men and women $(6.6 \mathrm{~kg}, 5.6 \%$ and $5.7 \mathrm{~kg}, 5.6 \%$, respectively, $\mathrm{p}=0.23$ ).

Patients were divided in tertiles according to the measured parameters of insulin resistance (Matsuda-Index, HOMA-Index and C-Peptide levels), age and BMI. $\bullet$ Table 2 and $\bullet$ Fig. 1 show the results. Both, absolute and relative weight loss increased with higher degrees of insulin resistance as measured by Matsuda-index and HOMA-index. However, weight loss was not associated with different levels of C-peptide. There was significantly more absolute weight loss with increasing BMI, but no significant association of relative weight loss with BMI. Moreover, age was unrelated to the degree of weight loss.

In the subgroup of patients with normal HOMA-index $(<2.5$, $\mathrm{n}=43$ ) the average weight loss was $4.9 \pm 8.9 \mathrm{~kg}$. In the subgroup of patients with normal HOMA- and normal Matsuda-index (>6; $\mathrm{n}=14$ ) the average weight loss was $5.3 \pm 8.9 \mathrm{~kg}$.

\section{Discussion \\ $\nabla$}

In our study we found an average weight loss of $5.8 \mathrm{~kg}(5.6 \%)$ under treatment with metformin for 6 months in overweight and obese, mostly insulin-resistant patients.

To our knowledge, this is the first study that assesses the effectiveness of metformin for weight-loss in a natural endocrine outpatient setting with particular regard to the degree of insulin resistance.

Although metformin is widely recognized by endocrinologists and diabetologists as a weight reducing agent in clinical practice, there is little evidence in the literature up to date supporting this empiric knowledge.

Even in recently published reviews on anti-obesity drugs metformin is not mentioned $[19,20]$. One review analysing studies describing weight changes under metformin came to the conclusion that there is not sufficient evidence to recommend metformin as a treatment of overweight or grade 1 obesity [33]. Importantly however, in that review, the duration of treatment in the majority of the examined studies was too short to see effects on weight (15 days to 3 months in 6 out of 9 studies), most of the studies were not designed to investigate weight reduction and the BMI measurements of study participants were limited to overweight (BMI $25-29.9 \mathrm{~kg} / \mathrm{m}^{2}$ ) or grade I obesity (BMI $30-34.9 \mathrm{~kg} / \mathrm{m}^{2}$ ), therefore severe insulin resistance in these patients was unlikely.

One randomized controlled study of high quality and 2 randomized controlled studies of fair quality investigated the efficacy of metformin as a weight reducing agent: in subjects at risk for the development of diabetes (Diabetes Prevention Program, base line weight $94.4 \mathrm{~kg}$ ), in subjects with an elevated waist to hip ratio (base line BMI 33.4) and in women suffering from PCOS (base line weight $97.0 \mathrm{~kg}$ ) [15-18]. A limited or insignificant weight reducing effect of metformin therapy was reported in these studies (Diabetes Prevention Program 2,7 kg, Fontbonne et al. and Gamberini et al. no significant effect). The weight loss induced by metformin treatment in our study of non-diabetic patients exceeds the amount of weight reduction reported by the above mentioned studies.

One reason may be that in the study of Gambineri at al., who did not observe a significant weight loss as compared to placebo, patients were already on a hypocaloric diet before metformin treatment was started. It is possible that a hypocaloric diet diminishes insulin resistance and thereby the effect of metformin [34]. Another reason maybe dosage of metformin. As we increased the dose of metformin depending on the weight of the patient up to $2500 \mathrm{mg}$ per day, other authors used lower doses. In the BigPro1 trial only $850 \mathrm{mg}$ of metformin had been administered daily to the study participants, possibly explaining the insignificant weight reduction in this study [17].

Moreover, none of these studies was conducted under natural outpatient circumstances. In a smaller open-label study in an 
outpatient-setting a weight loss of $6 \mathrm{~kg}$ was described in severely obese patients with mostly elevated fasting insulin levels (base line weight $117.3 \mathrm{~kg}$ ), an effect that was similar to our observation [35].

The controversial results in these studies may be largely caused by different degrees of insulin resistance in the examined study populations. As we could show in our study that insulin resistant patients lost more weight under metformin as compared to insulin sensitive patients, a turkish study described an average weight loss of $9 \mathrm{~kg}$ examining patients with pronounced insulin resistance (baseline weight $96 \mathrm{~kg}$; average HOMA-index 4,99) [36]. Unfortunately this study lacked an untreated control group, but compared metformin to orlistat and sibutramine, describing metformin as equally effective as orlistat.

Another study observed in obese type 2 diabetes patients - insulin resistant by definition - a weight loss of $8 \mathrm{~kg}$ after metformin treatment over 24 weeks [8].

Mogul et al. reported in 25 of 26 hyperinsulinemic, severly obese women (refractory to diet) a weight loss of at least $5 \%$ within the first 6 month of metformin therapy [37]. The hyperinsulinemia of these patients indicates the presence of insulin resistance and it is possible that this population may be more sensitive to effects of metformin on body weight.

Altogether, the discussed data suggest that the efficacy of metformin to reduce weight depends - at least partially - on the degree of insulin resistance.

It has been shown that insulin-receptor binding, the tyrosine kinase activity of the insulin receptor and glucose transport are altered in the same way in type 2 diabetes patients and comparably obese individuals without diabetes, which supports the efficacy of metformin in both conditions [38].

Today, the strong link between weight gain and insulin resistance is undisputed. There is compelling evidence that insulin resistance is a major contributor to abdominal obesity [10-12]. Yet, it hasn't been clarified if insulin resistance leads to or is rather a consequence of obesity [39].

Improving insulin resistance mediates weight loss by the following mechanisms:

First of all, improved insulin sensitivity reduces the frequency of postprandial hypoglycaemia which is caused by sluggish postprandial insulin secretion with a delayed insulin peak [40]. Less postprandial hypoglycaemic events result in less carbohydrate cravings and as a consequence in less compensatory food intake, especially of carbohydrates [41].

Moreover, metformin not only improves blood glucose control by insulin-stimulated glucose disposal in the skeletal muscle, but also decreases hepatic glucose output, inhibits gluconeogenesis and decreases intestinal glucose absorption from the gastrointestinal tract. [9], reviewed by [42]. These mechanisms provide less glucose for energy storage in the adipose tissue. Notably, it has been shown that, in contrast to weight loss induced by hypocaloric diet, metformin diminishes adipose tissue but not lean body mass [9].

Furthermore, abdominal fat tissue tends to retain its sensitivity to insulin in the face of hepatic and skeletal muscle resistance, which leads typically to a predominant increment of abdominal fat tissue in insulin resistant obese patients $[43,44]$. Restoring peripheral insulin sensitivity by metformin reduces pancreatic hypersecretion of insulin. As a consequence the insulin receptor of abdominal fat tissue is exposed to lower levels of insulin, which may result in a lower energy uptake by abdominal fat and thereby reduce abdominal fat mass.
Interestingly however, metformin did also cause significant weight loss in obese patients without insulin resistance (normal HOMA- or Matsuda-index). This leads to the postulation that other mechanisms than reducing insulin resistance by metformin contribute to weight loss in metformin treated patients. In the treatment of PCOS patients metformin has also be shown to be effective on oligomenorrhea and hyperandrogenemia in some patients despite the lack of insulin resistance [45].

Metformin leads to a decrease in appetite $[9,41]$ and certain authors discuss that metformin contains a primary anorectic factor [46]. One other reason may be a decrease of leptin levels, which has been observed under metformin treatment in adipose tissue in vivo as well as in serum levels in vivo suggesting an underlying improvement of leptin resistance $[47,48]$. Furthermore GLP-1 levels seem to rise significantly under metformin and may thus promote weight loss $[49,50]$.

In our study the amount of weight loss correlated with body weight at base line. It is evident that patients with severe obesity have a greater capacity to loose weight than patients with relatively mild obesity. Severely obese patients lost on average more weight as compared to patients with slighter obesity. However there was no significant difference in the percentage of weight reduction as compared to baseline under metformin in the higher BMI tertiles as compared to the lowest tertile. We also observed that the effectiveness of metformin as a weight reducing agent was independent of age or sex.

It is a limitation of our study that the control group was not randomized. Possibly the motivation to loose weight was different in this group. Also we only analyzed a follow-up period of 6 months. We do not know with certainty if these effects will endure. However the follow-up of the Diabetes Prevention Program points to a sustained effect of metformin, which is in line with our experience (data not shown) [10].

A strength of our study is that it shows the effect of metformin in a real life situation, and not in a clinical trial situation, including effects of compliance and potential interactions with comedications, among others. Additionally, we carefully assessed the association of insulin resistance with weight loss.

Taken together, our data suggest that if metformin is administered to obese patients in high enough doses it is a beneficial and cost effective drug to reduce weight. The effectiveness of metformin as a weight reducing agent is not restricted to insulin resistant patients although a greater weight loss can be expected if patients exhibit biochemical signs of insulin resistance prior to metformin treatment.

\section{Acknowledgement \\ $\nabla$}

We thank our study nurse Mrs Karin Binder for assistance in collection of the data and her help to follow up the patients

\section{Statement of conflict of interest: Nothing to declare.}

\section{References}

1 Li Z, Maglione M, Tu W et al. Meta-analysis: pharmacologic treatment of obesity. Ann Intern Med 2005; 142: 532-546

2 Christopoulou FD, Kiortsis DN. An overview of the metabolic effects of rimonabant in randomized controlled trials: potential for other cannabinoid 1 receptor blockers in obesity. J Clin Pharm Ther 2011; 36: $10-18$ 
3 Meneghini LF, Orozco-Beltran D, Khunti $K$ et al. Weight beneficial treatments for type 2 diabetes. J Clin Endocrinol Metab 2011; 96: 3337-3353

4 Bennett WL, Odelola OA, Wilson LM et al. Evaluation of guideline recommendations on oral medications for type 2 diabetes mellitus: a systematic review. Ann Intern Med 2012; 156: 27-36

5 Sterne J. Pharmacology and mode of action of hypoglycaemic guanidine derivatives. In: Campbell GD. (ed.). Oral hypoglycaemic agents. London: Academic, 1969; 193-245

6 Dunaif $A$. Insulin resistance and the polycystic ovary syndrome: mechanism and implications for pathogenesis. Endocr Rev 1997; 18: 774-800

7 Unlühizarci K, Keleştimur F, Bayram $F$ et al. The effects of metformin on insulin resistance and ovarian steroidogenesis in women with polycystic ovary syndrome. Clin Endocrinol 1999; 51: 231-236

8 Lee A, Morley JE. Metformin decreases food consumption and induces weight loss in subjects with obesity with type II non-insulin-dependent diabetes. Obes Res 1998; 6: 47-53

9 Stumvoll M, Nurjhan N, Perriello $G$ et al. Metabolic effects of metformin in non-insulin-dependent diabetes mellitus. N Engl J Med 1995; 333: 550-554

10 Lustig $R H$. Hypothalamic obesity: the sixth cranial endocrinopathy. The Endocrinologist 2002; 12: 210-217

11 Sigal RJ, El-Hashimy M, Martin BC et al. Acute post-challenge hyperinsulinemia predicts weight gain. Diabetes 1997; 46: 1025-1029

12 Odeleye OE, de Courten M, Pettitt DJ et al. Fasting hyperinsulinemia is a predictor of increased body weight gain and obesity in Pima Indian children. Diabetes 1997; 46: 1341-1345

13 Bailey DJ, Turner RC. Metformin. N Engl J Med 1996; 334: 574-579

14 Hermann LS, Melander A. Biguanides: basic aspects and clinical uses. In: Alberti KGMM, DeFronzo RA, Keen H, Zimmet P (eds.). International textbook of diabetes mellitus. Vol. 1: Chichester, England: John Wiley, 1992; 773-795

15 Knowler WC, Barrett-Connor E, Fowler SE et al. Reduction in the incidence of type 2 diabetes with lifestyle intervention or metformin. $\mathrm{N}$ Engl J Med 2002; 346: 393-403

16 Diabetes Prevention Program Research Group. Long-term safety, tolerability, and weight loss associated with metformin in the Diabetes Prevention Program Outcomes Study. Diabetes Care 2012; 35: 731-772

17 Fontbonne A, Diouf I, Baccara-Dinet $M$ et al. Effects of 1-year treatment with metformin on metabolic and cardiovascular risk factors in non-diabetic upper-body obese subjects with mild glucose anomalies: a post-hoc analysis of the BIGPRO1 trial. Diabetes Metab 2009; 35: 385-391

18 Gambineri A, Patton L, Vaccina $A$ et al. Treatment with flutamide, metformin, and their combination added to a hypocaloric diet in overweight-obese women with polycystic ovary syndrome. J Clin Endocrinol Metab 2006; 91: 3970-3980

19 Derosa G, Maffioli P. Anti-obesity drugs: a review about their effects and their safety. Expert Opin Drug Saf 2012; 11: 459-471

20 Ara R, Blake L, Gray L et al. What is the clinical effectiveness and cost-effectiveness of using drugs in treating obese patients in primary care? A systematic review. Health Technol Assess 2012; 1: 1-195

21 Bray GA, Ryan DH. Medical Therapy for the Patient with Obesity. Circulation 2012; 125: 1695-1703

22 Gillies CL, Abrams KR, Lambert PC et al. Pharmacological and lifestyle interventions to prevent or delay type 2 diabetes in people with impaired glucose tolerance: systematic review and meta-analysis. BMJ 2007; 334: 299-308

23 Setter SM, Iltz JL, Thams J et al. Metformin hydrochloride in the treatment of type 2 diabetes mellitus: a clinical review with a focus on dual therapy. Clin Ther 2003; 25: 2991-3026

24 Haffner S, Temprosa M, Crandall J et al. Intensive lifestyle intervention or metformin on inflammation and coagulation in participants with impaired glucose tolerance. Diabetes 2005; 54: 1566-1572

25 Wang $M$, Tong $\mathrm{JH}$, Zhu G et al. Metformin for treatment of antipsychotic-induced weight gain: A randomized, placebo-controlled study. Schizophr Res 2012; 138: 54-57

26 Bushe CJ, Bradley AJ, Doshi S et al. Changes in weight and metabolic parameters during treatment with antipsychotics and metformin: do the data inform as to potential guideline development? A systematic review of clinical studies. Int J Clin Pract 2009; 63: 1743-1761

27 Park MH, Kinra S, Ward KJ et al. Metformin for obesity in children and adolescents: a systematic review. Diabetes Care 2009; 32: 1743-1745
28 Mauras N, DelGiorno C, Hossain J et al. Metformin use in children with obesity and normal glucose tolerance-effects on cardiovascular markers and intrahepatic fat. J Pediatr Endocrinol Metab 2012; 25: 33-40

29 Metwally M, Amer S, Li TC et al. of metformin versus orlistat for the management of obese anovulatory women. Hum Reprod 2009; 24: 966-975

30 Harborne LR, Sattar N, Norman JE et al. Metformin and weight loss in obese women with polycystic ovary syndrome: comparison of doses. J Clin Endocrinol Metab 2005; 90: 4593-4598

31 Ghandi S, Aflatoonian A, Tabibnejad $N$ et al. The effects of metformin or orlistat on obese women with polycystic ovary syndrome: a prospective randomized open-label study. J Assist Reprod Genet 2011; 28: 591-596

32 Matsuda $M$, DeFronzo $R A$. Insulin sensitivity indices obtained from oral glucose tolerance testing: comparison with the euglycemic insulin clamp. Diabetes Care 1999; 22: 1462-1470

33 Levri KM, Slaymaker E, Last A et al. Metformin as treatment for overweight and obese adults: a systematic review. Ann Fam Med 2005; 3: 457-461

34 Goodpaster BH, Kelley DE, Wing RR et al. Effects of weight loss on regional fat distribution and insulin sensitivity in obesity. Diabetes 1999; 48: 839-847

35 Glueck CJ, Fontaine RN, Wang $P$ et al. Metformin reduces weight, centripetal obesity, insulin, leptin, and low-density lipoprotein cholesterol in nondiabetic, morbidly obese subjects with body mass index greater than 30. Metabolism 2001; 50: 856-861

36 Gokcel A, Gumurdulu Y, Karakose $H$ et al. Evaluation of the safety and efficacy of sibutramine, orlistat and metformin in the treatment of obesity. Diabetes Obes Metab 2002; 4: 49-55

37 Mogul HR, Peterson SJ, Weinstein BI et al. Long-term (2-4 year) weight reduction with metformin plus carbohydrate-modified diet in euglycemic, hyperinsulinemic, midlife women (Syndrome W). Heart Dis 2003; 5: 384-392

38 Dohm GL, Tapscott E, Pories W et al. An in vitro human muscle preparation suitable for metabolic studies. Decreased insulin stimulation of glucose transport in muscle from morbidly obese and diabetic subjects. J Clin Invest 1988; 82: 486-494

39 Oppert JM, Nadeau A, Tremblay A et al. Plasma glucose, insulin, and glucagon before and after long-term overfeeding in identical twins. Metabolism 1995; 44: 96-105

40 Luyckx AS, Lefevbre PJ. Plasma insulin in reactive hypoglycemia. Diabetes $1971 ; 20$ : 435-442

41 Schultes B, Oltmanns KM, Kern $W$ et al. Modulation of hunger by plasma glucose and metformin. J Clin Endocrinol Metab 2003; 88: 1133-1141

42 Giannarelli $R$, Aragona M, Coppelli A et al. Reducing insulin resistance with metformin: the evidence today. Diabetes Metab 2003; 29: 28-35

43 Mizuno TM, Funabashi T, Kleopoulos SP et al. Specific preservation of biosynthetic responses to insulin in adipose tissue may contribute to hyperleptinemia in insulin-resistant obese mice. J Nutr 2004; 134 : 1045-1050

44 Cusin I, Terrettaz J, Rohner-Jeanrenaud $F$ et al. Hyperinsulinemia increases the amount of GLUT4 mRNA in white adipose tissue and decreases that of muscles: a clue for increased fat depot and insulin resistance. Endocrinology 1990; 127: 3246-3248

45 Tan S, Hahn S, Benson S et al. Metformin improves polycystic ovary syndrome symptoms irrespective of pre-treatment insulin resistance. Eur J Endocrinol 2007; 157: 669-676

46 Paolisso G, Amato L, Eccellente $R$ et al. Effect of metformin on food intake in obese subjects. Eur J Clin Invest 1998; 28: 441-446

47 Mick GJ, Wang X, Ling Fu C et al. Inhibition of leptin secretion by insulin and metformin in cultured rat adipose tissue. Biochim Biophys Acta 2000; $1502: 426-432$

48 Morin-Papunen LC, Koivunen RM, Tomas $C$ et al. Decreased serum leptin concentrations during metformin therapy in obese women with polycystic ovary syndrome. J Clin Endocrinol Metab 1998; 83: 2566-2568

49 Mannucci E, Ognibene A, Cemasco F et al. Effect of metformin on glucagon-like peptide-1 (GLP-1) and leptin levels in obese nondiabetic subjects. Diabetes Care 2001; 24: 489-494

50 Mannucci E, Tesi F, Bardini $G$ et al. Effects of metformin on glucagonlike peptide-1 levels in obese patients with and without Type 2 diabetes. Diabetes Nutr Metab 2004; 17: 336-342 